\title{
Electrode for Wearable Electrotherapy ${ }^{\dagger}$
}

\author{
Meijing Liu *, Steve Beeby and Kai Yang \\ School of Electronics and Computer Science, University of Southampton, Southampton SO17 1BJ, UK; \\ spb@ecs.soton.ac.uk (S.B.); ky2@ecs.soton.ac.uk (K.Y.) \\ * Correspondence: ml5y17@soton.ac.uk \\ + Presented at the International Conference on the Challenges, Opportunities, Innovations and Applications \\ in Electronic Textiles (E-Textiles 2019), London, UK, 12 November 2019.
}

Published: 4 December 2019

\begin{abstract}
An electrode is a fundamental element used in many electrotherapy devices. This work presents a novel dry electrode made from carbon and silicone rubber materials for wearable electrotherapy applications. The electrode was mixed using a speed mixer and fabricated using stencil printing. This paper investigates the resistivity change of the electrode under the pressure from $0 \mathrm{mmHg}$ to $32.4 \mathrm{mmHg}$; and the skin-electrode impendence with the current frequency from $20 \mathrm{~Hz}$ to $10,000 \mathrm{~Hz}$. The resistivity of the novel dry electrode is $24.6 \pm 1.5 \Omega \cdot \mathrm{m}$ when the pressure on electrode is $17.7 \mathrm{mmHg}$. The skin-electrode impedance reduced from $1001.6 \Omega$ to $145.3 \Omega$ when the frequency increased from $20 \mathrm{~Hz}$ to $10,000 \mathrm{~Hz}$.
\end{abstract}

Keywords: osteoarthritis; E-textile; wearable; electrotherapy; stencil printing; electrode

\section{Introduction}

Osteoarthritis (OA) is a leading cause of disability in older adults which not only affects people's life quality (e.g., pain, sleep difficulties, depression) [1], but also represents a burden to the society [2]. In the UK, the cost of OA has been estimated to account for between $1 \%$ and $2.5 \%$ of the gross national product [3]. Pain is the main symptom for OA patients [4]. Electrotherapy has been used to reduce pain and improve physical functions.

Electrotherapy is used in pain relief by applying low current $(0-100 \mathrm{~mA})$ to the skin to stimulate the sensory nerves and interfere the pain signal transmission. There are mainly two types of electrotherapy, Transcutaneous Electrical Nerve Stimulation (TENS) and Interferential Therapy (IFT). The typical frequency range is $2-150 \mathrm{~Hz}$ for TENS and $1 \mathrm{~K}-10 \mathrm{~K} \mathrm{~Hz}$ for IFT. Positive results in pain relief have been achieved by both of them [5].

The electrotherapy devices include two parts: the electronic control unit to generate stimulus current and electrodes to transfer the current through the skin to stimulate sensory nerves. Traditional electrotherapy devices use gel electrodes which are not compatible with textiles. Gel electrodes are recommended to keep in sealed bags after use to maintain the moisture level. Even though, the standard lifetime for the gel electrodes are only 1-2 weeks due to the drying out of the electrode and the contamination caused by their sticky property. Moreover, in order to get the optimized performance, individual gel electrodes need to be positioned accurately which is timeconsuming. Improving the property of electrode has become a significant topic in the research of wearable electrotherapy devices.

This work presents a novel dry electrode which has been integrated into textile for wearable applications [6]. The electrode is soft and tacky providing good conformability with the skin. This paper investigates the electrical property under different pressure which is relevant to tightness of the clothing item (e.g., knee, sleeve) for wearable applications. The skin-electrode impedance is measured under the full range of electrotherapy frequency. 


\section{Materials}

The novel dry electrode paste is made from Fabink E-0002 (Smart Fabric Inks, Southampton, UK). It consists of a two-part silicone rubber and a conductive carbon black. These three ingredients are mixed at $3500 \mathrm{rmp}$ for $30 \mathrm{~s}$ using a speed mixer (SpeedMixerTM (DAC 150.1 FV)). The paste is placed in a $5 \mathrm{~cm} \times 5 \mathrm{~cm} \times 2 \mathrm{~mm}$ aluminium frame and casted using a squeegee to form a smooth surface (Figure 1a). The electrode together with the frame is cured at $80^{\circ} \mathrm{C}$ for $30 \mathrm{~min}$. Tearing off the cured material from the frame, a carbon-based electrode is fabricated (Figure 1b).

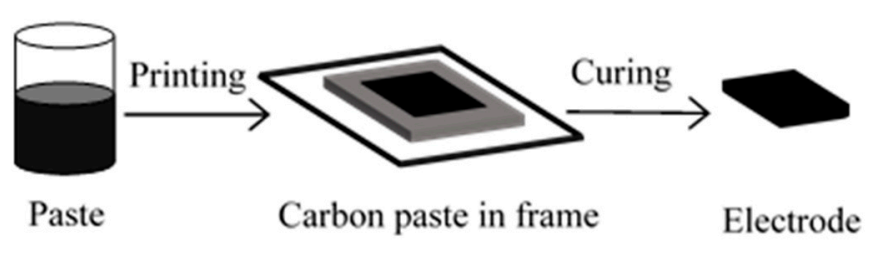

(a)

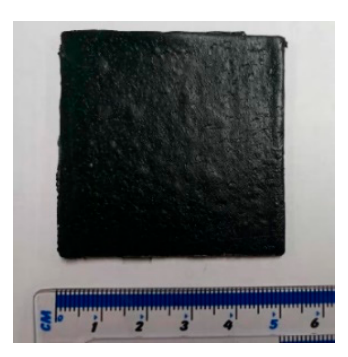

(b)

Figure 1. (a) The schematic diagram for stencil printing; (b) Dry electrode made from Fabink E-0002.

\section{Methods}

\subsection{Resistivity Measurement under Different Pressure}

In order to mimic the current (from the electrode top surface to the bottom) flow, the electrode is sandwiched between two copper layers and the resistance of the electrode is measured using a multimeter (FLUKE 115). A $5 \mathrm{~cm} \times 5 \mathrm{~cm}$ aluminium plate, together with a weight bar, is placed on the top of the copper layer to achieve even weight/pressure distribution on the electrode (Figure 2). The pressure adding on the electrode varies from $0 \mathrm{mmHg}$ to $32.4 \mathrm{mmHg}$ at $2.94 \mathrm{mmHg}$ intervals.

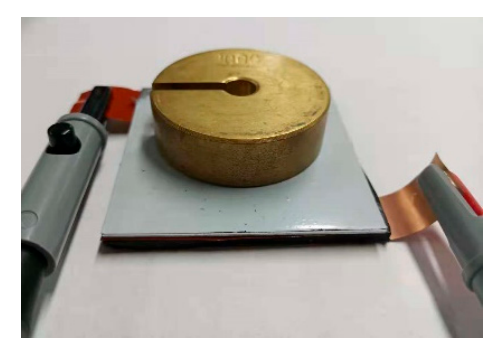

Figure 2. Electrode sandwiched by two copper layers with an aluminium plate and $2.94 \mathrm{mmHg}$ pressure on the top. The probes of multimeter are connected with the wings of the copper layers.

The resistivity is calculated by an equation:

$$
\rho=\mathrm{R} \cdot \mathrm{S} / \mathrm{L}
$$

where $R$ represents the resistance measured by the multimeter. $S$ and $L$ represent the surface area (5 $\mathrm{cm} \times 5 \mathrm{~cm})$ and the thickness $(2 \mathrm{~mm})$ of the electrode.

\subsection{Skin-Electrode Impedance Measurement under Different Frequency}

The impedance of skin-electrode is measured using a Precision Impedance Analyser 6500B supplied by Wayne Kerr as shown in Figure 3a. A piece of boneless pork belly joint is used to represent the human skin. Two electrodes are placed on the top of the skin with $5 \mathrm{~cm}$ distance in between. The probes of the analyser are connected with copper sheets which are attached on the top of electrodes. Water was applied on the electrode before it was placed on the skin. Figure $3 \mathrm{~b}$ has shown an example of the measurement setup. The frequency varies from $20 \mathrm{~Hz}$ to 10,000 $\mathrm{Hz}$. The 
impedance under different frequency is benchmarked against a commercial gel electrode (Axelgaard, PLAS) commonly used in electrical stimulation.

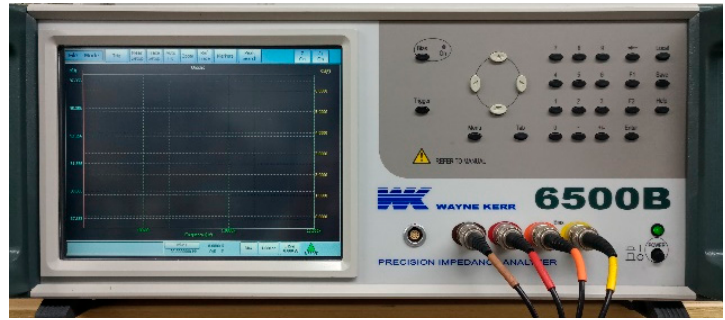

(a)

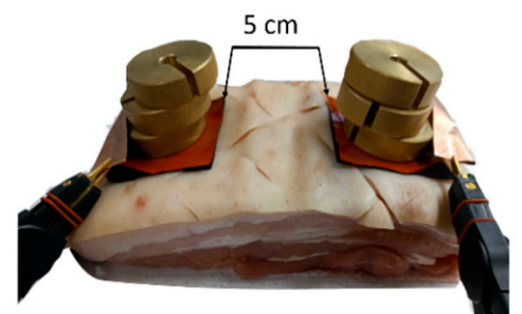

(b)

Figure 3. (a) The Precision Impedance analyser; (b) Two electrodes are placed on a piece of pork, with $8.8 \mathrm{mmHg}$ pressure on top of each electrode.

\section{Results and Discussion}

Figure 4 shows the resistivity of electrode decreased sharply when the pressure was increased from $0 \mathrm{mmHg}(72.2 \Omega \cdot \mathrm{m})$ to $5.9 \mathrm{mmHg}(40.4 \Omega \cdot \mathrm{m})$, followed by a slower decreasing when the pressure was further increased from $5.9 \mathrm{mmHg}$ to $17.7 \mathrm{mmHg}(24.6 \Omega \cdot \mathrm{m})$. The resistivity only changed $6 \Omega \cdot \mathrm{m}$ when the pressure increased from $17.7 \mathrm{mmHg}$ to $32.4 \mathrm{mmHg}$. The error bar of the resistivity was also deceased with the pressure which indicates the electrode performance became more uniform.

Adding pressure on the electrode can reduce the resistivity of the electrode and the variation on electrode performance, which can potentially reduce the power consumption and improve battery life. However, the pressure is limited by the safety and comfort in the wearable applications and the recommended value is less than $20 \mathrm{mmHg}$ [7]. Therefore, $17.7 \mathrm{mmHg}$ pressure is chosen for later research.

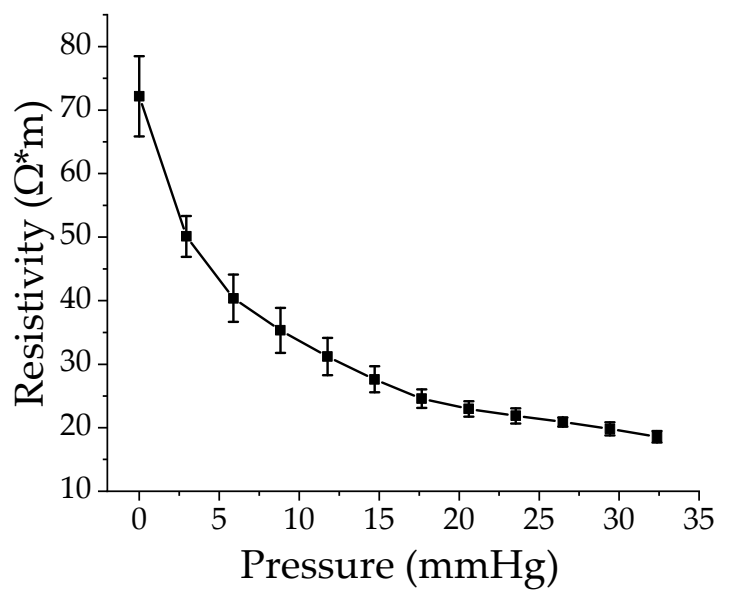

Figure 4. The resistivity of the electrode under different pressure.

Figure 5 shows the relationship between the impedance of skin-electrode and the frequency. The impedance of the skin-dry electrode is less than the skin-gel electrode throughout the measured frequencies. For TENS, which is typically around $100 \mathrm{~Hz}$, the impedance of skin-dry electrode is 520 $\Omega$. For IFT, typically around $4000 \mathrm{~Hz}$, the impedance is $169 \Omega$. For electrotherapy devices, the impedance of skin-electrode can guide the design of the electronic circuit for impedance/current matching. 


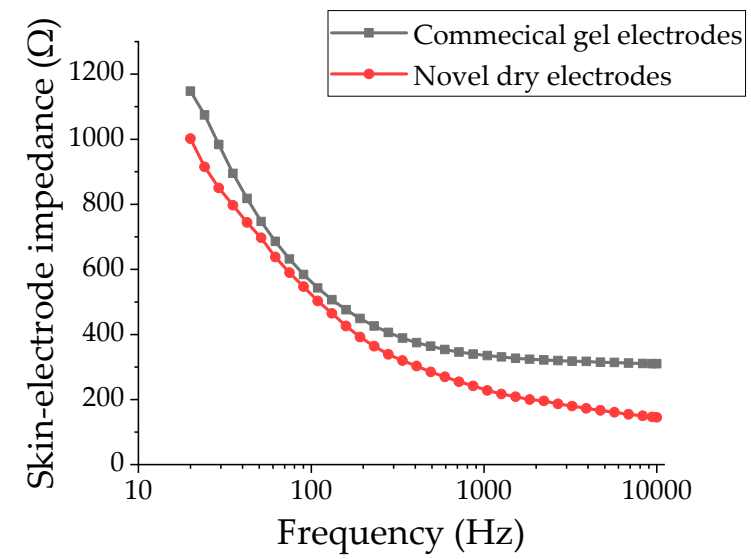

Figure 5. The impedance of skin-electrode with novel dry electrodes and commercial gel electrodes.

\section{Conclusions}

This work has demonstrated the fabrication of a dry electrode suitable for the wearable electrotherapy. The electrode resistivity and the error bar reduce with the increasing of the pressure applied on top of the electrode. A resistivity of $24.6 \pm 1.5 \Omega \cdot \mathrm{m}$ was achieved under the optimal pressure of $17.7 \mathrm{mmHg}$. The skin-electrode impedance of the electrode is lower than the commercial gel electrode. The measurement will be used to guide the electronic design.

Author Contributions: M.L. carried out the experiment design and implementation, and wrote the manuscript draft. K.Y. provided supervision for the overall research work and edited the manuscript. S.B. co-supervised the research.

Funding: This research was funded by EPSRC, grant number EP/S001654/1.

Conflicts of Interest: K.Y. and S.B. are directors of Smart Fabric Inks.

\section{References}

1. Allen, K.; Golightlya, Y.M. Epidemiology of osteoarthritis: State of the evidence. Curr. Opin. Rheumatol. 2015, 27, 276-283.

2. Arden, N.; Nevitt, M.C. Osteoarthritis: Epidemiology. Best Pract. Res. Clin. Rheumatol. 2006, 20, 3-25.

3. Hunter, D.J.; Schofield, D.; Callander, E. The individual and socioeconomic impact of osteoarthritis. Nat. Rev. Rheumatol. 2014, 10, 437-441.

4. Hunter, D.J.; McDougall, J.J.; Keefe, F.J. The Symptoms of Osteoarthritis and the Genesis of Pain. Rheum. Dis. Clin. N. Am. 2008, 34, 623-643.

5. de Almeida, C.C.; da Silva, V.Z.M.; Durigan, J.L.Q. Transcutaneous electrical nerve stimulation and interferential current demonstrate similar effects in relieving acute and chronic pain: A systematic review with meta-analysis. Braz. J. Phys. Ther. 2018, 22, 347-354.

6. Yang, K.; Meadmore, K.; Freeman, C.; Grabham, N.; Hughes, A.M.; Wei, Y.; Torah, R.; Glanc-Gostkiewicz, M.; Beeby, S.; Tudor, J. Development of User-Friendly Wearable Electronic Textiles for Healthcare Applications. Sensors 2018, 18, 2410.

7. Xiong, Y.; Tao, X. Compression garments for medical therapy and sports. Polymers (Basel) 2018, 10, 1-19. 